\title{
Short-term recognition memory under rehearsal instructions and imaging instructions
}

\author{
C. ALAN BONEAU \\ George Mason University, Fairfax, Virginia
}

\begin{abstract}
A modified Sternberg (1966) procedure with target sets of 3 to 18 food names was used with instructions either to rehearse items or to form a visual image of them. Under rehearsal instructions, omission errors (failures to recognize probed items that had been in the target set) were predominant; under imaging instructions, a small number of errors were made both for probed items in and not in the target set. A pronounced serial position effect emerged under rehearsal instructions, but only a small recency effect was evident under the imaging instructions. Thus results with rehearsal seem compatible with a buffer model, but imaging results do not.
\end{abstract}

Studies of short-term memory have utilized a variety of experimental procedures (e.g., Miller, 1956; Peterson \& Peterson, 1959; Sternberg, 1966), and these have utilized materials that lend themselves to a maintenance rehearsal (Craik \& Lockhart, 1972) process. Even when more meaningful stimulus materials are used, the memory process of rehearsal is usually presumed by default to be in effect.

As an alternative to rehearsal, the creation of visual images as a process to aid memory has been studied in a variety of contexts, but almost invariably with a focus on retention for longer periods of time. Explicitly visual material has also been used as stimuli, and a substantial number of pictures can be recognized over long periods of time (Shepard, 1967), the conclusion being that the process underlying this capability is automatic (Hasher \& Zacks, 1979). No experiments have been designed explicitly to study the effects of the imaging process on shortterm memory to compare the results to those obtained with a maintenance rehearsal procedure.

This experiment was designed to make such a comparison. A modification of the Sternberg (1966) paradigm, using target sets composed of single-word food names, was developed as a recognition paradigm analogous to the ordinary digit-span procedure. Items from target sets of varying lengths were exposed sequentially for 1 sec each. Probes either in or not in the target set were then used to test for recognition. It was hypothesized that instructions to create a visual mental image of the food items would result in better recognition than instructions to rehearse them by using overt vocalization. Also, since recognition procedures elicit a larger store of information than a recall procedure does, it was expected that the results from both procedures would provide evidence that

This paper is based on a similarly titled poster that was presented by the author at the convention of the American Psychological Society, Alexandria, VA, on June 12, 1989. Requests for reprints may be sent to C. Alan Boneau, at the following address: Department of Psychology, George Mason University, Fairfax, VA 22030. short-term memory "contains" substantially more items than Miller's (1956) "magical number seven."

\section{METHOD}

Target sets consisting of strings of single-word food names were sequentially presented on a variety of Apple II computer monitors for $1 \mathrm{sec}$ each, with no break between items. Two seconds later, a sequence of five probe words was given, with the words spaced approximately $6 \mathrm{sec}$ apart. The probes were displayed for up to $2 \mathrm{sec}$, in which time the subject was required to make a response, thereby terminating the display. The word "correct" was displayed for $.5 \mathrm{sec}$ for correct responses only when a feedback option was in effect. The subject's judgment of whether the probe stimulus was in or not in the target set and the subject's time to make this response were recorded for each probe. Also recorded were the serial positions of the probed items that were in the target set and, for the last several sessions, the number of the trial on which each probe item had last appeared in a target set. Target set lengths of $3,6,9,12,15$, or 18 items were selected randomly for each trial, with each length being represented equally often. The appropriate number of target items was then randomly selected on each trial from a pool of 50 such items. Probes were randomly selected on each trial, with probes in and probes not in the target set equally likely. Between trials, a variable delay averaging about $10 \mathrm{sec}$ occurred. A sequence of 30 trials constituted a session. Data from 24 sessions for each instruction condition are presented in this paper.

Under the rehearsal instructions, the subject repeated aloud strings of items from the sequence of stimulus words. Under the imaging instructions, the subject formed a visual image of each stimulus food item during its presentation. On the first 14 sessions, feedback was given for every correct response. On the final 10 , no feedback was given. A minimum of $.5 \mathrm{~h}$, and in many instances several days, occurred between sessions. The subject was seated comfortably with eyes approximately $2 \mathrm{ft}$ from the computer monitor.

The author served as the subject for the data presented here.

\section{RESULTS}

Under both instruction conditions, a relatively high but gradually declining level of correct responses was maintained for all target set lengths, as is shown in Figure 1. The imaging results are significantly higher than the rehearsal results $(p<.001)$. When correct responses for probes in or not in the target set are looked at separately, a major difference appears between the two instructions (see Figure 2). When the probed item was in the target 


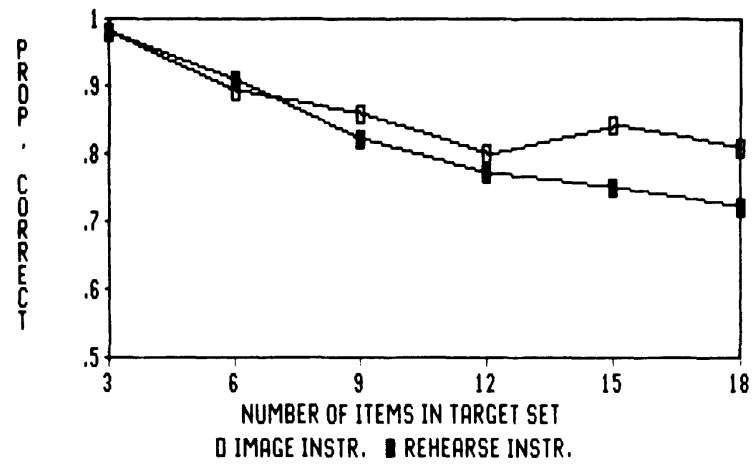

Figure 1. Proportion of correct responses for the several target set lengths, showing all probes for rehearsal and imaging instructions.

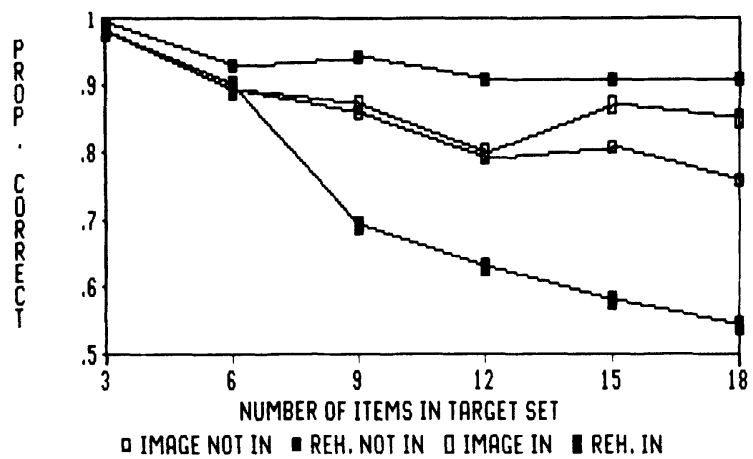

Figure 2. Proportion of correct responses for the several target set lengths showing in and not in items separately for rehearsal and imaging instructions.

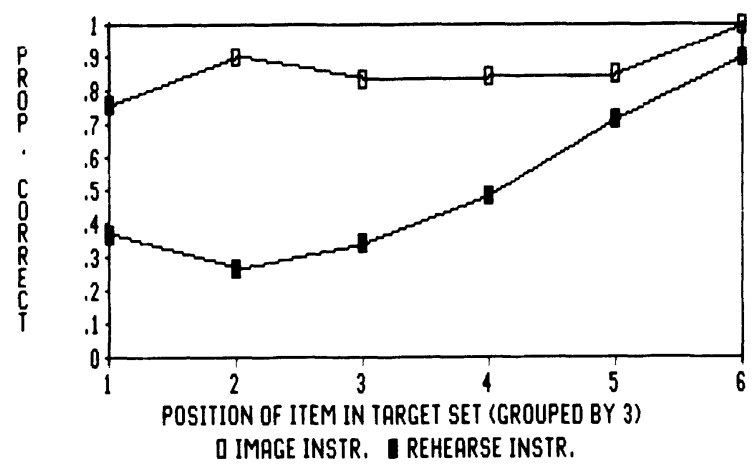

Figure 3. Serial position function showing proportion of correct responses for items at various serial positions (grouped by threes) for rehearsal and imaging instructions.

set, a relatively large number of errors (omission errors) occurred under the rehearsal instructions, with performance declining from nearly perfect for target sets of 3 , to nearly chance for sets of 18 . For not in probes, the level of correct responding remains fairly constant at about .90 , as can be seen in Figure 2. For the imaging instructions, the level of correct responding can be seen to be about the same for both conditions, declining from near perfect to the high .70 s. This interaction was significant $(p<.001)$.

Data for target set lengths of 18 for probed items in the target set were further analyzed to determine the probability of correct recognition when the probed food name was in various serial positions in the target set sequence. These data, shown in Figure 3 for stimulus positions grouped in threes, reveal a typical serial position function for the rehearsal instructions. In this condition, the proportion correct on the first three points is significantly less than chance $(p=.01)$. In data pertinent to the consideration of a primacy effect, however, the first point is not significantly higher than the other of the two.

In contrast, the data for the imaging instructions reveal what appears to be a minor recency effect, indicated by perfect performance when the probe item had been in one of the last three positions of the 18. Correct responding for the other three positions is essentially flat but at a high level for all positions.

Analysis of the data for probes not in the target set under the imaging instructions yielded information that a substantial number of errors occurred because the probed food item had been in the immediately prior target set but not the current set, being in the prior target set twice as often as would have occurred by chance.

Data not shown here revealed that under the rehearsal instructions, performance on the last two probes was significantly poorer $(p<.05)$ than on the first two when probed items were in the target set. Performance under the imaging instructions was virtually identical for all probes for both in and not in probes.

\section{DISCUSSION}

Traditional analyses of these data would make use of a signal-detection model (Egan, 1958). Accordingly, the relatively better performance under imaging instructions would reflect a smaller value of $d^{\prime}$ for this condition, whereas the interaction shown in Figure 2 could be attributed to differences in bias for the two instructions. Without ad hoc add-on to the signal-detection model, however, a number of details of the present data would not be readily explicable. Specifically, unadorned signaldetection theory has difficulty with the serial position results and with other details of the imaging data, all presented below.

The rehearsal data are consistent with a buffer model (see, e.g., Atkinson \& Shiffrin, 1968). According to this model, a small rehearsal buffer is searched. A subject's response indicates whether or not the probe item is found among the items in the buffer. For probes in the target set, the probability that the probed item will be retained in the subject's buffer decreases with target set size. This is because a buffer of fixed size will encompass smaller proportions of the items in the set. For not in probes, the probed item is never in the buffer by definition. Because of interference by probe items, performance should worsen with an increasing number of probes as intervening probes dislodge additional items from the buffer. The buffer model of Atkinson and Shiffrin (1968) is compatible with all of this and predicts the recency effect (and the small primacy effect) that can be seen in Figure 3.

The imaging data are inconsistent with the buffer predictions. Under these instructions, probe in and not in results are comparable and considerably better overall than are the results under the rehearsal instructions. The serial position function shows only a gradual decline with increasing distance from the final stimulus item. There is no decrease in performance across probes. I hypothesize that the imaging instructions call forth judgments about the recency of presentation of the probed 
item. Consistent with this hypothesis is the fact that an inordinate number of errors seem to have been made when the probed item was not in the present set, but was in the immediately prior set.

As for how many items are in short-term memory, a routine analysis used with true-false questions with a correction for guessing would, when applied to the 18-item data from Figure 1, yield approximately 9 "known" items under the rehearsal and 13 under the imaging instructions.

The data from this study suggest that the two kinds of instructions employed may bring different kinds of cognitive processes into play. The rehearsal data seem explicable in terms of a buffer model, but the imaging data do not. The imaging data suggest that the judgment that a probed item was in the target set may be at least in part a judgment that the item has been activated recently. The details of the data that suggest these different processes are not readily explicable with a simple signal-detection model. Additional research will be required to ascertain the validity of the hypothesis of two separate processes and to determine the limits of applicability of the signal-detection model.

\section{REFERENCES}

Atkinson, R. C., \& Shiffrin, R. (1968). Human memory: A proposed system and its control processes. In K. W. Spence \& J. T. Spence
(Eds.), The psychology of learning and motivation (Vol. 2, pp. 89195). Orlando, FL: Academic Press.

Craik, F. I. M., Lockhart, R. S. (1972). Levels of processing: A framework for memory research. Journal of Verbal Learning \& Verbal Behavior, 11, 671-684.

EGAN, J. B. (1958). Recognition memory and the operating characteristic (Technical Note AFCR-TN58-51). Bloomington: Indiana University Hearing and Communications Laboratory.

HASHER, L., \& ZACKS, R. T. (1979). Automatic and effortful processes in memory. Journal of Experimental Psychology: General, 108, 356-388.

Miller, G. A. (1956). The magical number seven, plus or minus two: Some limits on our capacity for processing information. Psychological Review, 63, 81-97.

Peterson, L. R., \& Peterson, M. J. (1959). Short-term retention of individual verbal items. Journal of Experimental Psychology, 58, 193-198.

SHEPARD, R. N. (1967). Recognition memory for words, sentences, and pictures. Journal of Verbal Learning \& Verbal Behavior, 6, 156-163. STERNBERG, S. (1966). High speed scanning in human memory. Science, 153, 652-654.

(Manuscript received September 29, 1989.) 